\title{
Percepção do impacto emocional da equipe de enfermagem diante da pandemia de COVID-19: relato de experiência
}

\author{
Perception of the emotional impact of the nursing staff in the face of the COVID-19 \\ pandemic: experience report
}

Percepción del impacto emocional del personal de enfermería ante la pandemia de COVID-19: informe de experiencia

\begin{abstract}
Jéssica Karoline Alves Portugal ${ }^{1 \star}$, Marcelo Henrique da Silva Reis ${ }^{1}$, Évelyn Janaína da Silva Barão ${ }^{1}$, Tanny Thaylle Gomes de Souza ${ }^{1}$, Ramanda Sena Guimarães ${ }^{1}$, Lucas da Silva de Almeida ${ }^{1}$, Rhuana Maria de Oliveira Pereira ${ }^{1}$, Nathalie Marinho Freire ${ }^{1}$, Sibele Naiara Ferreira Germano ${ }^{1}$, Marlucia da Silva Garrido'.
\end{abstract}

\section{RESUMO}

Objetivo: Relatar a percepção da equipe de enfermagem de um hospital em um município no interior do estado do Amazonas, diante da pandemia de coronavírus, expondo os principais medos e anseios frente as incertezas do novo cenário mundial e os desafios vindouros. Relato de experiência: Este relato é baseado na vivencia da equipe de enfermagem diante da pandemia do coronavírus, demonstrando seus medos e anseios e como os atuais acontecimentos no Brasil e no mundo podem afetar significativamente as condições psicológicas dos profissionais de saúde, uma vez que possuem maior risco de contaminação devido o contato direto com pacientes infectados. Desta forma, observou-se que as equipes de enfermagem têm atuado com constate pressão psicológica, podendo causar ansiedade e até mesmo depressão. Considerações finais: Os profissionais sofrem as mesmas pressões psicológicas, principalmente pelo medo diante da incerteza das condições futuras, tendo em vista a proporção da pandemia. Portanto, é preciso que os profissionais de saúde sejam atendidos em suas inquietações e desamparos, visto que a regressão da pandemia depende disso.

Palavras chave: Infecções por coronavírus, Enfermagem, Saúde mental.

\begin{abstract}
Objective: To report the perception of the nursing team of a hospital in the municipality of Coari in the interior of the state of Amazonas, in the face of the coronavirus pandemic, exposing the main fears and anxieties in view of the uncertainties of the new world scenario and the challenges to come. Experience report: This 1 epor tis based on the experience of the nursing team in the face of the coronavirus pandemic, demonstrating their fears and anxieties and how the current events in Brazil and in the world can significantly affect the psychological conditions of health professionals, since they have increased risk of contamination due to direct contact with infected patients. Thus, it was observed that the nursing teams have acted with constant psychological pressure, which can cause anxiety and even depression. Final considerations: professionals suffer the same psychological pressures, mainly due to fear in view of the uncertainty of future conditions, in view of the proportion of the pandemic. Therefore, it is necessary that health professionals are attended to in their concerns and helplessness, since the regression of the pandemic depends on it.
\end{abstract}

Key words: Coronavirus infections, Nursing, Mental health.

${ }_{1}$ Universidade Federal do Amazonas (UFAM), Coari - AM. *E-mail: jessika.portugal@gmail.com

SUBMETIDO EM: 5/2020

ACEITO EM: 5/2020

PUBLICADO EM: 5/2020

REAS/EJCH | Vol. Esp. 46 | e3794 | DOI: https://doi.org/10.25248/reas.e3794.2020 Página 1 de 6 


\section{RESUMEN}

Objetivo: Informar sobre la percepción del equipo de enfermería de un hospital en la ciudad de Coari, en el interior del estado de Amazonas, frente a la pandemia de coronavirus, exponiendo los principales temores y ansiedades ante las incertidumbres del nuevo escenario mundial y los desafíos por venir. Informe de experiencia: Este informe se basa en la experiencia del equipo de enfermería frente a la pandemia de coronavirus, demostrando sus temores y ansiedades y cómo los eventos actuales en Brasil y en el mundo pueden afectar significativamente las condiciones psicológicas de los profesionales de la salud, ya que han mayor riesgo de contaminación debido al contacto directo con pacientes infectados. Por lo tanto, se observó que los equipos de enfermería han actuado con presión psicológica constante, lo que puede causar ansiedad e incluso depresión. Consideraciones finales: Los profesionales sufren las mismas presiones psicológicas, principalmente debido al miedo en vista de la incertidumbre de las condiciones futuras, considerando la proporción de la pandemia. Por lo tanto, es necesario atender a los profesionales de la salud en sus preocupaciones e impotencia, ya que la regresión de la pandemia depende de ello.

Palabras clave: Infecciones por coronavirus, Enfermería, Salud mental.

\section{INTRODUÇÃO}

No final de 2019, uma notícia espalhou-se pelo mundo trazendo medo a todas as nações. A descoberta de um vírus altamente contagioso colocou as principais lideranças políticas em estado de alerta. Em janeiro de 2020, este assunto passou a ser o principal tópico em todos veículos de informações. A partir de então, todos tiveram conhecimento do novo Coronavírus, causador da doença nomeada como COVID-19 (LUIGI R e SENHORAS EM, 2020).

O COVID-19 foi descoberto em 31 de dezembro de 2019 em Wuhan, China e disseminou-se rapidamente tornando-se uma pandemia (USHER K, et al., 2020). O vírus é altamente patogênico e causa infecções do trato respiratório como a Síndrome Respiratória Aguda Grave (SRAG) e pode levar ao óbito (GUIMARÃES HP et al., 2020, FIORATTI C, 2020). É transmitido através de contato direto com gotículas de saliva produzidas através da fala, tosse e espirros de um indivíduo contaminado. Além disso, pode-se contrair o vírus ao tocar o rosto (olhos, nariz e boca) após o contato com superfícies e objetos contaminados (FRANCO AG, et al., 2020).

$\mathrm{Na}$ maioria dos casos os sintomas podem ser brandos ou até mesmo imperceptíveis. No entanto, os indivíduos sintomáticos podem apresentar sintomas comuns, como: febre, tosse e dificuldade de respirar. Os sintomas graves apresentam quadros de pneumonia grave (TUÑAS ITC, et al., 2020), acometendo principalmente pacientes idosos, portadores de doenças pré-existentes, como hipertensão, diabetes, doenças respiratórias crônicas e pessoas imunocomprometidas (CARVALHO AP, et al). Porém, o coronavírus não faz distinção de classe econômica, fronteiras, idiomas e ideologias, pois tem o poder de afetar a todos, seja direta ou indiretamente (DALTRO M e BARRETO SEGUNDO JD, 2020).

O vírus pode ficar incubado por até 14 dias no hospedeiro humano, aumentando suas chances de transmissão mesmo antes de aparecer os primeiros sintomas. Além disso, após a morte da pessoa infectada, o vírus pode permanecer ativo por vários dias (BRASIL, 2020). Neste contexto, a maior preocupação em relação ao vírus está relacionada ao seu alto poder de disseminação, pois os números de pessoas infectadas podem crescer gradativamente em poucos dias (MACEDO YM, et al., 2020).

No Brasil, o primeiro caso de COVID-19 foi identificado em 26 de fevereiro de 2020. O paciente era procedente da cidade de São Paulo e havia retornado de uma viagem na Itália dias antes do diagnóstico. Em poucos dias outras pessoas foram diagnosticadas, demonstrando a rapidez de transmissão da doença. (MACEDO YM, et al., 2020). Em 13 de março de 2020 foi registrado o primeiro caso de COVID-19 no estado do Amazonas. Embora o governo tenha adotado medidas restritivas para prevenção da infecção da doença, em 13 de abril, um mês após o surgimento do primeiro caso, o Amazonas já registrava um total de 1.206 casos e 62 mortes. Entre os casos positivos diagnosticados até 13 de abril de 2020, haviam 193 pacientes 
internados, sendo 82 em Unidades de Terapia Intensiva (UTI). Além disso, outros 406 pacientes internados estavam aguardando confirmação do diagnóstico como casos suspeitos, sendo 351 em leitos clínicos e 55 em UTI (DINIZ C, 2020).

Em Manaus, capital do Amazonas, o hospital de referência para tratamento de pacientes com COVID-1919 já havia atingido sua capacidade máxima em poucas semanas e o governo anunciou o risco de colapso dos serviços de saúde por falta de leitos, profissionais e equipamentos (DINIZ C, 2020b). Com o acelerado aumento dos números de casos, o Ministério da Saúde declarou que o Amazonas estaria entre os estados de maior transição para a descontrolada transmissão de coronavírus (DINIZ C, 2020)

Poucas semanas após a descoberta do primeiro caso de coronavírus no mundo, a Organização Mundial da Saúde (OMS) declarou estado de emergência em saúde pública de relevância internacional (FERNANDES EG, et al., 2020; FRANCO AG, et al., 2020). Diante deste cenário mundial, algumas medidas passaram a ser adotas. Na tentativa de reduzir a propagação do vírus, diversos países iniciaram campanhas de isolamento social, restringindo a população em diversas áreas da vida cotidiana (USHER K, et al., 2020).

A nova realidade passou a fazer parte do cotidiano de milhares de pessoas espalhadas por todos os continentes. No entanto, enquanto o mundo se recolhe, existem aqueles que encaram diariamente o novo "inimigo" neste combate. São os profissionais de saúde que vão para os hospitais e se colocam na linha de frente no enfrentamento da COVID-19, arriscando suas vidas e vivenciando situações adversas que vão desde desgastes físicos devido às altas cargas de trabalho, até desgastes psicológicos em decorrência do medo de adquirir a doença, além de lidar com a perda de pacientes e colegas de profissão (THE LANCET, 2020).

Os profissionais de saúde estão em alto risco de adquirir COVID-19 devido à exposição a pacientes e principalmente pela disponibilidade limitada de Equipamentos de Proteção Individual (EPI), falta de treinamento adequado para o enfrentamento de surtos de doenças altamente infecciosas (CHIEN CC e CHI CY, 2020) e uso inadequado de EPI (COOK TM, 2020).É imprescindível ressaltar que muitos profissionais de saúde foram infectados e vários perderam a vida desde o início da pandemia (LIMA CKT, et al.,2020).

Diante destes acontecimentos, as equipes de saúde estão sob constante pressão psicológica (CHEN Q, et al., 2020). Entre esses profissionais, destacam-se as equipes de enfermagem, tendo importante papel na saúde pública no controle e prevenção de infecção. Em todo mundo, a enfermagem tem trabalhado sob constante pressão, combatendo não apenas o vírus, mas diversas dificuldades impostas sobretudo pelo risco de infecção e pela escassez de proteção (SMITH GD, et al., 2020).

Diante do exposto, o objetivo deste estudo é relatar a percepção da equipe de enfermagem de um hospital em um município no interior do estado do Amazonas, diante da pandemia de Coronavírus, expondo os principais medos e anseios frente as incertezas do novo cenário mundial e os desafios vindouros.

\section{RELATO DE EXPERIÊNCIA}

Diante do surto mundial de COVID-19, a administração pública do município adotou medidas de contingenciamento já implementadas em vários territórios nacionais, determinando o fechamento de estabelecimentos comerciais não essenciais, como academias, centros de ginásticas e transporte de passageiros por via fluvial, além das suspensões das atividades escolares por tempo indeterminado.

No entanto, mesmo com todas as cautelas possíveis, no dia 10 de abril foi diagnosticado o primeiro caso de COVID-19 no município. Segundo informações, o paciente teria recentemente chegado de Manaus em uma embarcação. No dia seguinte, outro caso foi identificado no município. Desta forma, o risco que era iminente, passou a ser realidade entre a população coariense, gerando medo e incerteza sobretudo entre os profissionais de saúde, devido à insegurança e o despreparo diante da nova ameaça. Antes da confirmação dos casos, a secretaria municipal de saúde já preparava as equipes assistenciais para o adequado manejo de pacientes diagnosticados com COVID-19, disponibilizando treinamento, esclarecendo dúvidas acerca da doença e orientando quanto ao uso racional de equipamentos de proteção. Porém, conforme registrado em

REAS/EJCH | Vol. Esp. 46 | e3794 | DOI: https://doi.org/10.25248/reas.e3794.2020 Página 3 de 6 
vários locais do planeta, a escassez de EPI também se tornou um dos principais problemas enfrentados entre as equipes de saúde do município.

Entre as equipes de enfermagem atuantes no hospital, pode-se observar um considerável aumento na utilização de equipamentos de proteção. Porém, com a redução de distribuição desses materiais para diversos estados brasileiros, os profissionais de saúde, sobretudo enfermeiros e técnicos de enfermagem, passaram a sentir o impacto causado pelo coronavírus mesmo antes de sua chegada. Diante disto, o medo e insegurança que antecederam a chegada do vírus, colocaram as equipes de enfermagem em estado de alerta, em que muitos levantaram o seguinte questionamento: Estamos preparados para enfrentar um inimigo de tamanha magnitude?

Desta forma, a grande preocupação diante dos novos acontecimentos no Brasil e no mundo trouxe uma nova rotina para as equipes de enfermagem do serviço hospitalar, pois além de buscarem alternativas para se protegerem frente a escassez de EPI, também deveriam estar atentos para minimizar os riscos aos pacientes e acompanhantes dentro das unidades de internação. Neste contexto, o cuidado com a limpeza dos setores passou a ser redobrado, a utilização de máscaras, gorros, aventais e óculos de proteção que anteriormente eram utilizados apenas durante a realização de procedimentos, passaram a ser utilizados de forma ininterrupta, causando desconforto e irritação nos profissionais.

Porém, as equipes de enfermagem não passaram por mudanças apenas na sua rotina de trabalho. Podese observar que o medo relacionado ao risco de contágio hospitalar, levou muitos profissionais a adotarem medidas severas de cuidados pessoais no ambiente familiar. A maioria dos profissionais ressaltam que 0 maior receio seria uma possível transmissão do vírus para pessoas da família. Diante disto, a rotina familiar desses profissionais também foi alterada drasticamente, levando-os a se adaptarem a nova realidade de forma abrupta, evitando contato com pai, mãe, esposo (a) e filhos. Além disto, a única pausa do isolamento domiciliar passou a ser a rotina de trabalho hospitalar, onde deveriam lidar com conflitos maiores referentes ao controle de infecção e contato com pacientes com alto potencial de contágio.

Todas essas mudanças nos modos de vida tornaram esses profissionais mais ansiosos e inseguros, pois não se sabe quais proporções o vírus poderá atingir e o quanto isso irá afetar o município. Essa é uma preocupação relacionada não apenas ao número de pessoas que podem ser contaminadas, mas também quanto a segurança dos profissionais diante do enfrentamento da doença, uma vez que precisam atuar com proteção reduzida pela escassez de material.

Outro ponto importante, diz respeito ao preparo dos profissionais quanto à assistência a pacientes diagnosticados com COVID-19, pois muitos afirmam despreparo diante da nova doença e sentimento de incapacidade, visto que o tratamento é incerto e o risco de morte é elevado a depender o perfil do paciente. Portanto, o estado psicológico desses profissionais foi afetado significativamente, visto que passaram a experimentar situações adversas em seu ambiente de trabalho e convívio familiar em decorrência do novo e desconhecido problema enfrentado mundialmente.

Diante do exposto, nota-se que mesmo havendo poucos casos no município, a pandemia tem atingido consideravelmente a saúde de profissionais de assistência hospitalar, principalmente no que diz respeito aos aspectos psicológicos, devido as chances de exposição ocupacional, falta de equipamento de proteção, mudanças nas rotinas de trabalho e familiar e despreparo na assistência a pacientes infectados. Desta forma, é possível que pressão psicológica possa causar danos maiores do que o vírus propriamente dito.

Portanto, diante da grande turbulência emocional vivenciada pelas equipes de enfermagem, é necessário que sejam oferecidas medidas de proteção adequadas para esses profissionais, tendo em vista a redução da ansiedade ocasionada pelo medo de se contaminar. Além disso, o suporte psicológico é uma estratégia que pode ser implementada no hospital, a fim de minimizar a sobrecarga emocional nesse momento crítico.

\section{DISCUSSÃO}

Diante dos problemas emocionais enfrentados pelos profissionais durante a pandemia, Li Z, et al. (2020) explicam que os surtos de doenças infecciosas podem causar sentimentos de angústia e ansiedade, além de

REAS/EJCH | Vol. Esp. 46 | e3794 | DOI: https://doi.org/10.25248/reas.e3794.2020 Página 4 de 6 
traumatização direta e indireta entre os profissionais de saúde, devido às altas cargas de trabalho e falta de dispositivos de proteção. Quanto aos Equipamentos de Proteção Individual, existem dois problemas principais: Escassez e uso inadequado (COOK TM, 2020). Além de trabalhar em situações inseguras, esses profissionais sentem-se angustiados pela incerteza do tratamento clínico associada a imprecisão de informações a respeito da trajetória da pandemia e os resultados obtidos em curto e longo prazo (SMITH GD, et al., 2020).

Quanto ao desconforto relacionado ao uso constante de EPI, foi identificado que em vários lacais esse tem sido um problema comum. Em Wuhan, China, foi realizada uma pesquisa entre profissionais de saúde atuantes em hospitais de tratamento de COVID-19, onde houve relatos de reações adversas decorrentes do uso de EPI, destacando-se as dermatites, reações alérgicas, suor excessivo causado pelo uso prolongado de roupas e maceração na face. Além disso, muitos profissionais na China recorreram a métodos radicais, como uso de fraldas para reduzir as retiradas dos equipamentos diante as necessidades de ida ao banheiro e muitas enfermeiras cortaram o cabelo para permitir a colocação e retirada de roupas com mais facilidade, diminuindo os riscos de infecção (FABER M, 2020).

Em uma entrevista realizada com uma equipe médica de um hospital na China, muitos profissionais demonstraram sinais de irritabilidade, angústia e excitabilidade. Informaram que a maior preocupação não era serem infectados, mas sim levar uma possível infecção para suas famílias. Além disso, relataram que a faltam de colaboração por parte dos pacientes estava lhes causando maiores sofrimentos psicológicos, pois muitos não respeitavam as medidas preventivas de disseminação do vírus, consequentemente aumentando as chances de sobrecarregarem as unidades hospitalares. As equipes também informaram suas preocupações quanto a disponibilidade de material de proteção e a sensação de impotência diante de pacientes graves (CHEN Q, et al., 2020).

Lima CKT, et al. (2020), afirmam que a maioria dos profissionais de saúde que trabalham em unidade de isolamento não possuem treinamento adequado para lidar com problemas de saúde. Este fato corrobora com uma das aflições observadas nas equipes de enfermagem de Coari em relação ao despreparo profissional frente a gravidade dos pacientes. Na Itália, também é possível observar os impactos psicológicos causados pela pandemia, demonstrando altos níveis de estresse entre a população, ocasionado pela incerteza quanto ao tempo de progressão do surto, o medo de morrer e perder seus familiares nesse processo (LIMA CKT, et al., 2020).

Com o aumento de desgaste psicológico, é possível que sintomas psiquiátricos sejam intensificados em indivíduos com doença mental pré-existente (KELVIN DJ e RUBINHO S, 2020). Diante disto, é provável que as taxas de suicídio aumentem entre os profissionais de saúde em 2020. Segundo Goyal K, et al. (2020), foram identificados alguns casos de suicídios entre profissionais de saúde na Índia e na Itália que estavam infectados por COVID-19, comprovando que as consequências emocionais em decorrência da pandemia devem ser analisadas para adoção de estratégias que minimizem tais impactos.

Quanto a mudança drástica na rotina dos profissionais, é possível que cause diversas condições psicológicas. Ornell $\mathrm{F}$, et al. (2020), afirma que além do medo de morrer, a pandemia pode ocasionar mudanças em vários eixos, incluindo a rotina familiar, rotinas de trabalho, isolamento e fechamento de estabelecimentos como empresas e escolas. Além disso, o impacto econômico tem causado grande insegurança, gerando sentimento de desamparo e abandono.

Observa-se que os problemas enfrentados entre os profissionais de saúde durante a pandemia são semelhantes, independentemente da localização geográfica, da estrutura física e recursos humanos. Obviamente, não há comparação entre milhares de casos ocorridos na China e dois casos em um município pequeno.

No entanto, todos os profissionais sofrem as mesmas pressões psicológicas, principalmente pelo medo diante da incerteza das condições futuras, tendo em vista a proporção da pandemia em países desenvolvidos e no Brasil que possui menos recursos, onde a calamidade nos serviços de saúde já existe antes do coronavírus. Portanto, é preciso que os profissionais de saúde sejam atendidos em suas inquietações e desamparos, visto que a regressão da pandemia depende disso.

REAS/EJCH | Vol. Esp. 46 | e3794 | DOI: https://doi.org/10.25248/reas.e3794.2020 Página 5 de 6 


\section{REFERÊNCIAS}

1. BRASIL. Ministério da Saúde. Manejo de corpos no contexto do novo coronavírus COVID-19. Brasília: Ministério da Saúde, 2020.

2. CARVALHO AP, et al. Novo coronavírus (COVID-19). Sociedade Brasileira de Pediatria, Departamento científico de infectologia, 2020.

3. CHEN Q, et al. Mental Health care for medical staff in China during the COVID-19 outbreak. The Lancet Psychiatry, 2020.

4. CHIEN CC, CHI CY. Biosafety in the Preparation and Processing of Cytology Specimens With Potential Coronavirus (COVID-19) Infection: Perspectives From Taiwan. Cancer Cytopathology, 2020.

5. COOK TM. Personal protective equipment during the COVID-19 pandemic - a narrative review. Anaesthesia, 2020.

6. DALTRO M, SEGUNDO JDB. A pandemia que nos mostra quem somos? Revista Psicologia, Diversidade e Saúde, 2020; 9(1): 5-8.

7. DINIZ C. Há um mês, Amazonas registrava primeiro caso de Covid-19; Número passa de 1,2 mil, com mais de 60 mortes. G1 Amazonas.

8. DINIZ C. Hospital referência em Covid-19 de Manaus atinge capacidade máxima operacional, diz governo.

9. FARBER M. Coronavirus nurses in China are shaving their heads to 'improve efficiency'. Fox News.

10. FERNANDES EG, et al. Investigação do surto em navio de carga em tempo de COVID-19, Porto de Santos, Brasil. Revista Saúde Pública, 2020; 54(34): 1-4.

11. FIORATTI C. Sim, o coronavírus veio da natureza - e não de um laboratório. Revista Super Interessante. Disponível em: http://www.super.abril.com.br. Acesso em: 04/04/2020.

12. FRANCO AG, et al. Máscaras cirúrgicas em tempos de coronavírus. Interamerican jornal of medicine and health, 2020.

13. GOYAL K, et al. Fear of COVID 2019: First suicidal case in India! Asian J. Psychiatr, 2020; 49.

14. GUIMARÃES HP, et al. Coronavírus e Medicina de Emergência: Recomendações para o atendimento inicial do Médico Emergencista pela Associação Brasileira de Medicina de Emergencia (ABRAMEDE), 2020

15. KELVIN DJ, RUBINO S. Fear of the novel coronavirus. J. Infect. Dev. Ctries, 2020 14(1).

16. $\mathrm{LI} \mathrm{Z,} \mathrm{et} \mathrm{al.} \mathrm{Vicarious} \mathrm{traumatization} \mathrm{in} \mathrm{the} \mathrm{general} \mathrm{public,} \mathrm{members,} \mathrm{and} \mathrm{non-members} \mathrm{of} \mathrm{medical} \mathrm{teams} \mathrm{aiding} \mathrm{in}$ COVID-19 control. Brain Behav, 2020.

17. LIMA CKT, et al. O impacto emocional do Coronavírus 2019-nCoV (nova doença de Coronavírus). Psiquiatry Research, 2020; 287.

18. LUIGI R, SENHORAS EM. O novo coronavírus e a importância das Organizações Internacionais". Nexo Jornal $[17 / 03 / 2020]$.

19. MACEDO YM, et al. COVID - 19 NO BRASIL: o que se espera para população subalternizada? Revista Encantar Educação, Cultura e Sociedade - Bom Jesus da Lapa, 2020; 2: 01-10.

20. ORNELL F, et al. "Pandemic fear" and COVID-19: mental health burden and strategies. Brazilian Journal of Psychiatry, 2020.

21. REIS MHS, et al. Ações de saúde em populações ribeirinhas no interior do estado do Amazonas: Relato de experiência. Em SCHWEICKARDT JC. Atenção Básica na região amazônica: Saberes e práticas para o fortalecimento do SUS. Rede Unida, 2019; 171-181.

22. SMITH et al. COVID-19: Emerging compassion, courage and resilience in the face of misinformation and adversity. Journal of Clinical Nursing, 2020.

23. THE LANCET. COVID-19: protecting health-care workers. Lancet, 2020; 395: 922.

24. TUÑAS ITC, et al. Doença pelo Coronavírus 2019 (COVID-19): Uma abordagem preventiva para Odontologia. Revista Brasileira de Odontologia, 2020; 77: e 1766.

25. USHER K, et al. Life in the pandemic: Social isolation and mental health. J Clin Nurs, 2020. 